\title{
Differentiation between benign and malignant ovarian masses in the preoperative period using neutrophil-to-lymphocyte and platelet-to-lymphocyte ratios
}

\author{
MELAHAT YILDIRIM $^{1}$, BUSRA DEMIR CENDEK ${ }^{2}$ and AYSE FILIZ AVSAR ${ }^{3}$ \\ ${ }^{1}$ Department of Obstetrics and Gynecology, Ankara Ataturk Training and Research Hospital, Bilkent; \\ ${ }^{2}$ Department of Obstetrics and Gynecology, Sincan Dr. Nafiz Korez State Hospital, Sincan; \\ ${ }^{3}$ Department of Obstetrics and Gynecology, Yildirim Beyazit University, Bilkent, Ankara, Turkey
}

Received October 1, 2014; Accepted December 20, 2014

DOI: $10.3892 / \mathrm{mco} .2014 .481$

\begin{abstract}
The aim of this study was to evaluate the association between preoperative neutrophil-to-lymphocyte ratio (NLR) and platelet-to-lymphocyte ratio (PLR) and the pathological diagnosis of adnexal masses. The predictive effect of inflammatory markers on epithelial ovarian cancer was also investigated. The present study included a total of 306 patients with adnexal masses who underwent surgical resection and the diagnosis was based on pathological investigation. The patients were divided into six groups based on their pathological findings and compared with respect to their NLR and PLR values. We used receiver-operating characteristic curves to calculate optimal cut-off values for NLR and PLR to predict ovarian cancer preoperatively. Patients with ovarian cancer exhibited significantly higher NLR and PLR values $(\mathrm{P}<0.05$ and $\mathrm{P}<0.001$, respectively). The multivariate analysis demonstrated that higher NLR and PLR values predicted ovarian cancer at the cut-off value of 3.35 , sensitivity of $55 \%$ and specificity of $81 \%$ for NLR [95\% confidence interval (CI): 0.544-0.752, $\mathrm{P}<0.05]$ and at the cut-off value of 572.9 , sensitivity of $100 \%$ and specificity of $0.38 \%$ for PLR (95\% CI: 0.192-0.381, $\mathrm{P}=0.001)$. Therefore, preoperative NLR and PLR values may help identify ovarian cancer in patients with adnexal masses.
\end{abstract}

\section{Introduction}

Ovarian cancer is the fifth leading cause of cancer-related mortality among women and the most lethal gynecological malignancy worldwide (1). Ovarian cancer has a fairly poor prognosis due to the advance stage of the disease at the time of diagnosis $(2,3)$ and the survival rates of the patients have

Correspondence to: Dr Melahat Yildirim, Department of Obstetrics and Gynecology, Ankara Ataturk Training and Research Hospital, Bilkent, 06000 Ankara, Turkey

E-mail: melahatyildrim@yahoo.com

Key words: neutrophil-to-lymphocyte ratio, platelet-to-lymphocyte ratio, benign ovarian masses, ovarian cancers remained constant for decades due to this reason. There are no sensitive and specific markers for epithelial ovarian cancer, since the early stages of this disease are completely asymptomatic (4). In addition, it is very difficult to distinguish benign ovarian lesions from ovarian cancer preoperatively. It was previously demonstrated that inflammation is the main contributor to the development and spread of cancer in the human body $(5,6)$. Inflammatory response against tumor cells may lead to irreversible DNA damage by inhibiting apoptosis of the ovarian cancer cells and triggering angiogenesis. This process has been shown to allow the tumor to grow constantly, invade the nearby tissue and subsequently spread to other sites in the body (5-7). Furthermore, growth factors released from platelets, such as platelet-derived growth factor, transforming growth factor $\beta$ and vascular endothelial growth factor, may also contribute to the growth and the development of the tumor $(8,9)$. Thrombocytosis is also associated with poor prognosis due to the increase in the number of platelets, leading to the increase in the growth factors released from these cells (10).

Preoperative inflammatory markers, such as neutrophil-to-lymphocyte ratio (NLR) and platelet-to-lymphocyte ratio (PLR), have been the subject of numerous studies on cancer (11-14) and it has been proven that inflammatory markers may reach significant levels in the blood in different types of cancer $(15,16)$. Therefore, it was hypothesized that these aforementioned preoperative inflammatory markers, which are known to be cost-effective and universally accepted, may help distinguish between malignant and benign ovarian tumors prior to undergoing surgery. The aim of this study was to determine inflammatory markers with a high predictive rate in order to differentiate between benign vs. malignant ovarian masses during the preoperative period.

\section{Patients and methods}

Patients. This retrospective comparative study included 306 patients with adnexal pathology. All the participants underwent surgical operation due to adnexal masses between January, 2007 and December, 2013 and their diagnosis was confirmed by pathological assessment. The study protocol was 
approved by the Ethics Committee of Ankara Ataturk Training and Research Hospital. Subsequently, the demographic and clinicopathological characteristics of patients with adnexal masses were investigated and recorded. The preoperative complete blood count values of patients were also measured and recorded for this study.

Adnexal mass classification and staging. The patients were divided into six different groups based on their pathological diagnosis of adnexal masses [72 patients with simple ovarian cysts, 66 with ovarian endometrioma, 42 with dermoid cysts, 79 with benign ovarian tumors (serous cystadenoma, mucinous cystadenoma), 40 with ovarian epithelial cancer and 7 with borderline ovarian tumors]. The type and stage of epithelial ovarian cancer were classified using the TNM system and the guideline of the National Cancer Institute of the USA (17). Since almost all the patients with ovarian epithelial cancer exhibited advanced-stage disease (stage IIIc or IV), we were unable to further classify ovarian cancer into subgroups based on stage. Dermoid cysts were classified as a different group from benign ovarian tumors, due to their easily recognizable and distinguishable ultrasound images or magnetic resonance imaging characteristics.

Preoperative assessment and patient classification. The preoperative complete blood count (CBC) parameters of the patients, such as white blood cell count, hemoglobin ( $\mathrm{Hgb})$, hematocrit, neutrophil count, lymphocyte count, monocyte count, NLR, platelet count, PLR, red cell distribution width (RDW), platelet distribution width (PDW) and mean platelet volume, were recorded and evaluated for each of the six aforementioned groups. Subsequently, all the patients were classified into two groups based on the diagnosis of ovarian cancer or lack thereof and a group-wise comparison was performed with respect to $\mathrm{CBC}$ parameters to detect any differences.

Statistical analysis. The recommended cut-off value of the preoperative variables that predict ovarian cancer was established using receiver operating characteristic (ROC) curve analyses. The optimal cut-off value of the NLR, PLR and RDW was based on the most prominent point on the ROC curve for sensitivity and specificity. The normality of distribution for variables was assessed using the Shapiro-Wilk test. Data are presented as means \pm SD for continuous variables with normal distribution and as median and interquartile range for continuous variables without normal distribution. To assess the differences in variables among groups, the Kruskal-Wallis test was used, followed by evaluation with the Mann-Whitney $\mathrm{U}$ test for multiple comparisons. The resulting P-values were corrected according to the Bonferroni method. ROC curves were used for the sensitivity and specificity of defined variables, such as NLR and PLR, to distinguish ovarian cancer from benign lesions. The area under the curve (AUC) and 95\% confidence intervals for defined variables were estimated. In addition to calculating the sensitivity and the specificity of the aforementioned variables, the positive and negative predictive values of these parameters were also calculated for distinguishing ovarian cancer. All the statistical analyses were performed using the Statistical Package for Social Sciences software, version 21.0 (IBM Corp. Released 2012. IBM
SPSS Statistics for Windows, Armonk, NY, USA). A P-value of $<0.05$ was considered to indicate statistically significant differences.

\section{Results}

Comparison of specified variables with defined groups. In the present study, the median age (IQR) for the groups was distributed as 46 (25) years for the simple ovarian cyst group, 39 (14) years for the endometrioma group, 36.50 (22) years for the dermoid cyst group, 51 (20) years for the benign ovarian tumor group, 63.50 (23) years for the ovarian epithelial cancer group and 61 (26) years for the borderline ovarian tumor group. The comparison of specified variables with defined groups in this study is shown in Table I.

The median NLR values were calculated as 2.09 (1.42) for the simple ovarian cyst group, 2.33 (1.56) for the endometrioma group, 2.01 (0.95) for the dermoid cyst group, 2.25 (1.26) for the benign ovarian tumor group, 3.54 (2.41) for the ovarian epithelial cancer group and $2.34(0.92)$ for the borderline ovarian tumor group (Table I). The differences in NLR values among defined groups were found to be statistically significant $\left(\chi^{2}=12.217, \mathrm{P}<0.05\right)$. The differences in PLR values among groups were also found to be statistically significant in this study $\left(\chi^{2}=21.649, \mathrm{P}=0.001\right)$. The median PLR values were found to be 125.89 (72.55), 140.22 (65.8), 134.4 (59.8), 128.08 (78.5), 198.87 (145.7) and 134.80 (73.7), in the order of the abovementioned groups (Table I). Although the PLR values were the lowest in the simple ovarian cyst group [125.89 (72.55)], they were found to be the highest in the ovarian epithelial cancer group [198.87 (145.7)]. PLR was calculated as 128.08 (78.5) for the benign ovarian tumor group, which was considerably different from the ovarian cancer group.

Briefly, NLR, Hgb, Hct, PDW and PLR were found to be significantly different among the six groups $(\mathrm{P}=0.032$, $\mathrm{P}=0.003, \mathrm{P}=0.011, \mathrm{P}=0.04$ and $\mathrm{P}=0.001$, respectively) (Table $\mathrm{I}$ ).

Comparison of variables exhibiting between-group differences. The individual significance of the difference in the aforementioned variables between two groups is presented in Table II. The statistically significant differences between the ovarian cancer and the other groups were obtained, in terms of defined variables (Table II). There were significant differences between the benign ovarian tumor group and the ovarian epithelial cancer group regarding NLR $(\mathrm{P}<0.05), \mathrm{Hgb}(\mathrm{P}<0.05)$ and PLR $(\mathrm{P}<0.001)$ values. The Hgb, Hct, and PLR values differed between the dermoid cyst and the ovarian epithelial cancer groups $(\mathrm{P}=0.007, \mathrm{P}<0.05$ and $\mathrm{P}<0.05$, respectively) (Table II). The only variable that was found to be statistically significant between the ovarian cancer and the remaining groups was $\mathrm{Hgb}(\mathrm{P}<0.05)$.

Comparison of defined variables between the benign tumor and the ovarian cancer groups. There were significant differences in the NLR values between the ovarian cancer and the benign tumor groups in this study [3.54 (2.41) vs. 2.25 (1.26); $\mathrm{P}<0.05]$. Similarly, the median PLR value was found to be statistically different between the ovarian cancer and the benign tumor groups [198.87 (145.71) and 128.08 (78.56), respectively; $\mathrm{P}<0.001]$ (Table III). 
ROC curve analyses. ROC curve analyses were performed to calculate the cut-off value of the variables. The recommended cut-off value of NLR in terms of predicting ovarian cancer was found to be 3.35 [sensitivity, 55\%; specificity, $81 \%$; positive predictive value (PPV), 30.98\%; and negative predictive value (NPV), 92.07\%] (Table IV). The AUC was $0.648 \pm 0.053$ (95\% CI: 0.544-0.752) ( $\mathrm{P}=0.003)$.

The recommended cut-off value for PLR was defined as 572.9 regarding identifying ovarian cancer preoperatively (sensitivity, 100\%; specificity, 0.38\%; PPV, 13.46\%; and NPV, 100\%) (Table IV). The AUC for PLR was $0.286 \pm 0.048$ (95\% CI: 0.192-0.381) $(\mathrm{P}<0.001)$. In the ROC analysis, performed based on the presence or absence of ovarian cancer, the AUC was found to be statistically significant for NLR and PLR values $\left(\mathrm{AUC}_{\mathrm{NLR}}=0.64\right.$ and $\left.\mathrm{AUC}_{\mathrm{PLR}}=0.28\right)(\mathrm{P}=0.003$ and $\mathrm{P}<0.001$, respectively).

\section{Discussion}

The present study included a total of 306 patients with adnexal masses and clearly demonstrated that preoperative NLR and PLR values were significantly higher in the ovarian cancer group compared to to those in the benign ovarian tumor group. The Hgb values were found to be the lowest in the ovarian cancer group in this study. This finding may be associated with the catabolic state of cancer. Since distinguishing between benign ovarian tumors (serous cystadenoma, mucinous cystadenoma) and epithelial ovarian cancer preoperatively is almost impossible, this significant finding may change the way the clinicians approach patients with adnexal masses. The recommended cut-off value of NLR in terms of predicting ovarian cancer was set at 3.35, with a sensitivity of $55 \%$ and a specificity of $81 \%$ in the ROC analysis. The optimal cut-off value for the PLR values was also calculated to be 572.9, with a sensitivity of $100 \%$ for identifying ovarian cancer during the preoperative period.

The association of preoperative NLR and PLR values with various cancer types has been the subject of numerous studies (12-15). All those studies also demonstrated that there is a significant correlation between preoperative NLR, PLR values and cancer stage, prognosis and response to treatment.

Absenger et al (16) demonstrated that patients with NLR $>4$ exhibited a longer median time-to-recurrence compared to patients with NLR $\leq 4$ during the treatment of colon cancer. In this study, increased NLR values were found to be significantly associated with shorter time-to-recurrence $(\mathrm{P}=0.001)$ in patients with stage II and III colon cancer. Furthermore, the study of Watanabe et al (18), evaluating the close association between inflammatory markers and ovarian cancer, revealed that preoperative inflammatory marker levels increase with disease progression. All these findings prove that there is a strong association of inflammation and immunosuppression with the natural immunity of cancer. Patients with advanced cancer usually exhibit increased neutrophil and decreased lymphocyte levels in the blood, allowing us to use NLR as one of the easiest and most effective markers of chronic inflammation and associated immune suppression in these patients $(19,20)$. Another study demonstrated that high serum inflammatory marker levels are an adverse prognostic factor for ovarian cancer (21). A previous study by Asher et al (22) clearly 
Table II. Comparison of variables exhibiting between-group differences among the six groups.

\begin{tabular}{lccccc}
\hline Type of tumor & $\begin{array}{c}\text { NLR } \\
\text { P-value }\end{array}$ & $\begin{array}{c}\text { Hgb } \\
\text { P-value }\end{array}$ & $\begin{array}{c}\text { HCT } \\
\text { P-value }\end{array}$ & $\begin{array}{c}\text { PDW } \\
\text { P-value }\end{array}$ & $\begin{array}{c}\text { PLR } \\
\text { P-value }\end{array}$ \\
\hline Dermoid cyst-benign tumor & NS & NS & NS & NS & NS \\
Dermoid cyst-simple cyst & NS & NS & NS & NS & NS \\
Dermoid cyst-endometrioma & NS & NS & NS & NS & NS \\
Dermoid cyst-borderline tumor & NS & NS & NS & NS & NS \\
Dermoid cyst-ovarian cancer & NS & 0.007 & $<0.05$ & NS & $<0.05$ \\
Benign tumor-simple cyst & NS & NS & NS & NS & NS \\
Benign tumor-endometrioma & NS & NS & NS & NS & NS \\
Benign tumor-borderine tumor & NS & NS & NS & $<0.05$ & NS \\
Benign tumor-ovarian cancer & $<0.05$ & $<0.05$ & NS & NS & $<0.001$ \\
Simple cyst-endometrioma & NS & NS & NS & NS & NS \\
Simple cyst-borderline tumor & NS & NS & NS & NS & NS \\
Simple cyst-ovarian cancer & NS & $<0.05$ & $<0.05$ & NS & 0.001 \\
Endometrioma-borderline tumor & NS & NS & NS & NS & NS \\
Endometrioma-ovarian cancer & NS & NS & NS & NS & $<0.05$ \\
Borderline tumor-ovarian cancer & NS & $<0.05$ & NS & NS & NS \\
\hline
\end{tabular}

NS, not significant; NLR, neutrophil-to-lymphocyte ratio; Hgb, hemoglobin; HCT, hematocrit; PDW, platelet distribution width; PLR, platelet-to-lymphocyte ratio.

Table III. Comparison of defined variables between the benign tumor and the ovarian cancer groups.

\begin{tabular}{lcccc}
\hline Variables & Benign tumor, median $(\mathrm{IQR})$ & Ovarian cancer, median $(\mathrm{IQR})$ & $\mathrm{Z}$ & P-value \\
\hline WBC $(\mathrm{K} / \mu \mathrm{l})$ & $7.83(2.79)$ & $7.57(2.25)$ & 0.453 & $\mathrm{NS}$ \\
NLR & $2.25(1.26)$ & $3.54(2.41)$ & 3.259 & 0.002 \\
HGB $(\mathrm{g} / \mathrm{dl})$ & $12.80(2.00)$ & $11.70(2.25)$ & 3.141 & 0.002 \\
HCT $(\%)$ & $37.90(5.60)$ & $34.80(5.35)$ & 2.734 & 0.006 \\
PDW (fl) & $14.40(3.65)$ & $12.60(5.60)$ & 2.873 & 0.004 \\
PLR & $128.08(78.56)$ & $198.87(145.71)$ & 4.213 & $<0.001$ \\
\hline
\end{tabular}

WBC, white blood cell count; NLR, neutrophil-to-lymphocyte ratio; HGB, hemoglobin; HCT, hematocrit; RDW, red blood cell distribution width; MPV, mean platelet volume; PDW, platelet distribution width; PLR, platelet-to-lymphocyte ratio; PLT, platelet; IQR, interquartile range; NS, not significant.

Table IV. Area under the receiver operating characteristic curve and cut-off values of NLR and PLR.

\begin{tabular}{lcccccccc}
\hline Variables & Cut-off & AUC \pm SE & 95\% CI & P-value & Sensitivity (\%) & Specificity (\%) & PPV (\%) & NPV (\%) \\
\hline NLR & 3.35 & $0.648 \pm 0.053$ & $0.544-0.752$ & 0.003 & 55.00 & 81.00 & 30.98 & 92.07 \\
PLR & 572.9 & $0.286 \pm 0.048$ & $0.192-0.381$ & $<0.001$ & 100.00 & 0.38 & 13.46 & 100.0
\end{tabular}

NLR, neutrophil-to-lymphocyte ratio; PLR, platelet-to-lymphocyte ratio; AUC, area under the curve; SE, standard error; CI, confidence interval; PPV, positive predictive value; NPV, negative predictive value.

demonstrated the association between NLR and PLR values and the prognosis of ovarian cancer. In that study, Asher et al observed that a high preoperative PLR value was significantly associated with a poor survival rate among epithelial ovarian cancer cases. While the median overall survival in patients with a PLR of <300 was 37.4 months (95\% CI: 26.1-48.7), in those with a PLR of $>300$ it was 14.5 months (95\% CI: 11.7-17.2) $(\mathrm{P}=0.03)$. Based on those findings, they concluded that PLR may be a novel independent prognostic marker in patients with ovarian cancer (22). 
Cho et al (23) investigated the diagnostic value of NLR in epithelial ovarian cancer cases and found that preoperative NLR in ovarian cancer patients (mean, 6.02) was significantly higher compared to that in benign ovarian tumor patients (mean, 2.57), benign gynecological disease patients (mean, 2.55) and healthy controls (mean, 1.98) $(\mathrm{P}<0.001)(20)$. Furthermore, they found the sensitivity and specificity of NLR in detecting ovarian cancer were $66.1 \%$ (95\% CI: 59.52-72.68\%) and $82.7 \%$ (95\% CI: 79.02-86.38\%), respectively (cut-off value, 2.60). In the present study, we reached results similar to those of the aforementioned study. In the present study, the median value of NLR was found to be 3.54 (2.41) in the ovarian cancer group, 2.09 (1.42) in the simple ovarian cyst group, 2.33 (1.56) in the endometrioma group and 2.25 (1.26) in the benign ovarian tumor group. The differences among groups were statistically significant $(\mathrm{P}<0.05)$. The sensitivity and specificity of NLR in detecting ovarian cancer, however, were found to be 55 and $81 \%$, respectively (cut-off value of NLR, 3.35; 95\% CI: 54.4-75.2\%). These findings were very similar to those of Cho et al (23). Another study demonstrated that high NLR values were closely associated with advanced cancer stage (24). Since the number of patients with ovarian cancer was limited and all the patients had advanced-stage disease, we were unable to evaluate the association between NLR, PLR and cancer stage. In addition, the limited number of patients with borderline ovarian tumors was the main limitation of this study, whereas our most significant finding was that malignant ovarian lesions may be distinguished from benign lesions using preoperative NLR and PLR values.

In conclusion, NLR and PLR are effective inflammatory markers in terms of predicting epithelial ovarian cancer preoperatively. These two cost-efeective and readily available markers may be used to support the diagnosis of ovarian cancer prior to undergoing surgery.

\section{References}

1. Jemal A, Siegel R, Ward E, Hao Y, Xu J and Thun MJ: Cancer statistics, 2009. CA Cancer J Clin 59: 225-249, 2009.

2. McGuire WP, Hoskins WJ, Brady MF, et al: Cyclophosphamide and cisplatin compared with paclitaxel and cisplatin in patients with stage III and stage IV ovarian cancer. N Engl J Med 334: $1-6,1996$

3. Markman M, Bundy BN, Alberts DS, et al: Phase III trial of standard-dose intravenous cisplatin plus paclitaxel versus moderately high-dose carboplatin followed by intravenous paclitaxel and intraperitoneal cisplatin in small-volume stage III ovarian carcinoma: an intergroup study of the Gynecologic Oncology Group, Southwestern Oncology Group and Eastern Cooperative Oncology Group. J Clin Oncol 19: 1001-1007, 2001.

4. Wei SH, Chen CM, Strathdee G, et al: Methylation microarray analysis of late-stage ovarian carcinomas distinguishes progression-free survival in patients and identifies candidate epigenetic markers. Clin Cancer Res 8: 2246-2252, 2002.

5. Jackson JR, Seed MP, Kircher CH, Willoughby DA and Winkler JD: The codependence of angiogenesis and chronic inflammation. FASEB J 11: 457-465, 1997.

6. Balkwill $\mathrm{F}$ and Mantovani A: Inflammation and cancer: back to Virchow? Lancet 357: 539-545, 2001.
7. Grivennikov SI, Greten FR and Karin M: Immunity, inflammation and cancer. Cell 140: 883-899, 2010.

8. Assoian RK and Sporn MB: Type beta transforming growth factor in human platelets: release during platelet degranulation and action on vascular smooth muscle cells. J Cell Biol 102: 1217-1223, 1986

9. Dubernard V, Arbeille BB, Lemesle MB and Legrand C: Evidence for an alpha-granular pool of the cytoskeletal protein alpha-actinin in human platelets that redistributes with the adhesive glycoprotein thrombospondin-1 during the exocytotic process. Arterioscler Thromb Vasc Biol 17: 2293-22305, 1997.

10. Allensworth SK, Langstraat CL, Martin JR, et al: Evaluating the prognostic significance of preoperative thrombocytosis in epithelial ovarian cancer. Gynecol Oncol 130: 499-504, 2013.

11. Unal D, Eroglu C, Kurtul N, Oguz A and Tasdemir A: Are neutrophil/lymphocyte and platelet/lymphocyte rates in patients with non-small cell lung cancer associated with treatment response and prognosis? Asian Pac J Cancer Prev 14: 5237-5242, 2013.

12. Feng JF, Huang Y, Zhao Q and Chen QX: Clinical significance of preoperative neutrophil lymphocyte ratio versus platelet lymphocyte ratio in patients with small cell carcinoma of the esophagus. Scientific World Journal 504365, 2013.

13. Wang D, Yang JX, Cao DY, Wan XR, Feng FZ, Huang HF, Shen K and Xiang Y: Preoperative neutrophil-lymphocyte and platelet-lymphocyte ratios as independent predictors of cervical stromal involvement in surgically treated endometrioid adenocarcinoma. Onco Targets Ther 6: 211-216, 2013.

14. Absenger G, Szkandera J, Pichler M, et al: A derived neutrophil to lymphocyte ratio predicts clinical outcome in stage II and III colon cancer patients. Br J Cancer 109: 395-400, 2013.

15. Cedrés S, Torrejon D, Martínez A, et al: Neutrophil to lymphocyte ratio (NLR) as an indicator of poor prognosis in stage IV non-small cell lung cancer. Clin Transl Oncol 14: 864-869, 2012.

16. Absenger G, Szkandera J, Stotz M, et al: Preoperative neutrophil-to-lymphocyte ratio predicts clinical outcome in patients with stage II and III colon cancer. Anticancer Res 33: 4591-4594, 2013.

17. National Cancer Institute of USA: Stage Information for Ovarian Epithelial Cancer. Available at: http://www.cancer.gov/cancertopics/pdq/treatment/ovarianepithelial/HealthProfessional/page3. Accessed December 19, 2014.

18. Watanabe T, Shibata M, Nishiyama H, et al: Serum levels of rapid turnover proteins are decreased and related to systemic inflammation in patients with ovarian cancer. Oncol Lett 7: 373-377, 2014.

19. McMillan DC, Canna K and McArdle CS: Systemic inflammatory response predicts survival following curative resection of colorectal cancer. Br J Surg 90: 215-219, 2003.

20. Pierce BL, Ballard-Barbash R, Bernstein L, et al: Elevated biomarkers of inflammation are associated with reduced survival among breast cancer patients. J Clin Oncol 27: 3437-3444, 2009.

21. Kodama J, Miyagi Y, Seki N, et al: Serum C-reactive protein as a prognostic factor in patients with epithelial ovarian cancer. Eur J Obstet Gynecol Reprod Biol 82: 107-110, 1999.

22. Asher V, Lee J, Innamaa A and Bali A: Preoperative platelet lymphocyte ratio as an independent prognostic marker in ovarian cancer. Clin Transl Oncol 13: 499-503, 2011.

23. Cho H, Hur HW, Kim SW, et al: Pre-treatment neutrophil to lymphocyte ratio is elevated in epithelial ovarian cancer and predicts survival after treatment. Cancer Immunol Immunother 58: 15-23, 2009.

24. KaoSC,Pavlakis N,Harvie R, Vardy JL,Boyer MJ,vanZandwijkN and Clarke SJ: High blood neutrophil-to-lymphocyte ratio is an indicator of poor prognosis in malignant mesothelioma patients undergoing systemic therapy. Clin Cancer Res 16: 5805-5813, 2010 . 